\title{
Expression and Functional Analysis of Dkk1 during Early Gonadal Development
}

\author{
A.N.Combes ${ }^{\text {a }}$ J. Bowles ${ }^{\text {a, b }}$ C.-W. Feng ${ }^{\text {a }}$ H.S.Chiu ${ }^{\text {a }}$ P.-L. Khoo ${ }^{c}$ A. Jackson ${ }^{a}$ \\ M.H. Little ${ }^{a}$ P.P.L. Tam ${ }^{c}$ P. Koopman ${ }^{a, b}$ \\ ${ }^{a}$ Division of Molecular Genetics and Development and ${ }^{b}$ ARC Centre of Excellence in Biotechnology and \\ Development, Institute for Molecular Bioscience, The University of Queensland, Brisbane, Qld., and \\ 'Embryology Unit, Children's Medical Research Institute, Sydney Medical School, University of Sydney, \\ Wentworthville, N.S.W., Australia
}

\section{Key Words}

Dkk1 $\cdot$ Rspo1 $\cdot$ Testis development $\cdot$ Wnt4

\begin{abstract}
WNT signalling plays a central role in mammalian sex determination by promoting ovarian development and repressing aspects of testis development in the early gonad. Dickkopf homolog 1 (DKK1) is a WNT signalling antagonist that plays critical roles in multiple developmental systems by modulating WNT activity. Here, we examined the role of DKK1 in mouse sex determination and early gonadal development. Dkk1 mRNA was upregulated sex-specifically during testis differentiation, suggesting that DKK1 could repress WNT signalling in the developing testis. However, we observed overtly normal testis development in Dkk1-null XY gonads, and found no significant upregulation of Axin2 or $S p 5$ that would indicate increased canonical WNT signalling. Nor did we find significant differences in expression of key markers of testis and ovarian development. We propose that DKK1 may play a protective role that is not unmasked by lossof-function in the absence of other stressors.
\end{abstract}

\section{KARGER}

Fax +41613061234

E-Mail karger@karger.ch

www.karger.com (c) 2011 S. Karger AG, Basel

Accessible online at: www.karger.com/sxd
Development of the bipotential mouse gonad into either a testis or an ovary depends on a transcriptional balancing act [Kim and Capel, 2006]. Testis development is triggered by SRY, the Y-chromosomal testis-determining factor, which needs to upregulate genes such as $\operatorname{Sox} 9$ and Fgf9 early enough and strongly enough to overcome the ovarian program of development. Conversely, the testicular program can be overcome to some extent by the actions of ovarian secreted factors RSPO1 (roof plate-specific spondin 1) and WNT4 (wingless-type MMTV integration site 4), both of which appear to function via activating the canonical $\beta$-catenin signalling pathway [reviewed by Tevosian and Manuylov, 2008]. Central to this competitive mode of sexual differentiation are factors that antagonise the opposing pathway to ensure faithful development of one gonadal fate, and avoid ambiguous outcomes such as ovotestes.

WNT signalling has emerged as a core module of the program regulating ovary development. In Wnt4-null XX mouse gonads, partial sex reversal is observed: müllerian ducts are absent, wolffian ducts are retained and the coelomic blood vessel, which is characteristic of the testis, forms ectopically [Vainio et al., 1999; Jeays-Ward et al., 2003; Heikkila et al., 2005]. In addition, ovary-associated transcripts Bmp2, Fst and Daxl are not expressed in Wnt4-knockout XX gonads, although expression of

Peter Koopman

Division of Molecular Genetics and Development, Institute for Molecular Bioscience The University of Queensland

Brisbane, QLD 4072 (Australia)

Tel. +61 733462059 , E-Mail p.koopman@imb.uq.edu.au 
Rspo1 is not affected [Jordan et al., 2001; Mizusaki et al., 2003; Yao et al., 2004; Chassot et al., 2008]. Curiously, ablation of Wnt4 delays testis development in XY gonads, perhaps reflecting an early role in the supporting cell lineage [Jeays-Ward et al., 2004]. Rspo1-null XX mouse gonads are also partially sex-reversed, with a phenotype similar to that of Wnt4-null XX gonads, although reportedly slightly more severe [Chassot et al., 2008; Tomizuka et al., 2008]. RSPO1 appears to be genetically upstream of WNT4, since early female-specific upregulation of Wnt4 expression is not observed in Rspo1-null gonads [Chassot et al., 2008; Tomizuka et al., 2008]. In humans, mutations in $R S P O 1$ have been shown to underlie cases of $\mathrm{XX}$ sex reversal [Parma et al., 2006], affirming the importance of WNT signalling to human ovarian development.

Mechanistically, WNT4 activates $\beta$-catenin remotely, by binding a Frizzled (Fz) receptor (one of the family of $\mathrm{Fz}$ serpentine receptors) and phosphorylating its co-receptor, low-density lipoprotein receptor-related protein 5 or 6 (LRP5/6), thereby triggering stabilization of cytosolic $\beta$-catenin, allowing its accumulation in the cytoplasm and subsequently facilitating translocation to the nucleus [He et al., 2004; Tamai et al., 2004; Davidson et al., 2005; Zeng et al., 2005]. In the nucleus, $\beta$-catenin complexes with TCF (T-cell factor)/LEF (lymphoid enhancer factor) transcription co-factors which activate the transcription of target genes. In the absence of nuclear $\beta$ catenin, TCF/LEF proteins recruit Groucho proteins and act conversely as transcriptional repressors.

DKK1 is a secreted antagonist of LRP6; DKK1 binds with high affinity to LRP6 and, together with its co-receptor Kremen, induces LRP6 endocytosis, thus effectively removing the LRP6 protein from the cell surface and making cells less able to respond to WNT and RSPO1. Although Kremen was thought to be integral to this process, a recent report has shown that DKK1 can also act in the absence of this protein [Ellwanger et al., 2008]. Loss of DKK1 frees the LRP6 from inhibition, resulting in enhanced WNT signalling activity. RSPO1, which also interacts with the LRP6 co-receptor, activates $\beta$-catenin signalling via phosphorylation of LRP6 [Nam et al., 2006; Wei et al., 2007], or by competitive binding to Kremen [Binnerts et al., 2007], thereby preventing the DKK1/Kremen-dependent internalization of LRP6. Whatever is the mechanism of action, evidence for DKK1 repression of $\mathrm{WNT} / \beta$-catenin signalling is extensive.

$D k k 1$ displays a sex-specific expression pattern in the developing gonad [Manuylov et al., 2008]. It is expressed initially in the developing gonad of both sexes at 11.5 days post coitum (dpc). $D k k 1$ is upregulated in the testis at 12.5 $\mathrm{dpc}$ and $13.5 \mathrm{dpc}$, whereas in the ovary, its expression is repressed by the GATA4-FOG2 transcription complex [Manuylov et al., 2008]. Despite the relatively low expression of $D k k 1$ in the developing ovary, $\beta$-catenin target genes $S p 5$ and $\operatorname{Ir} x 3$, as well as the key ovarian development gene Foxl2, were found to be upregulated in $D k k 1$ null XX gonads at $12.5 \mathrm{dpc}$ [Manuylov et al., 2008]. Because of the known function of DKK1 in dampening cellular response to WNT and RSPO1 signalling, we hypothesised that Dkk1-null XY gonads might be particularly sensitive to WNT4 and RSPO1 and therefore could become feminised to some extent. We studied $D k k 1$-null $\mathrm{XY}$ gonads at $11.5 \mathrm{dpc}$ and $12.5 \mathrm{dpc}$ time points and found no upregulation of canonical $\beta$-catenin pathway target genes. In addition, we found no apparent feminisation of the XY gonads in terms of marker gene expression and morphology. These results suggest that $D k k 1$ plays a backup or fail-safe role in preventing WNT signalling, rather than providing the primary means of repressing the ovarian pathway in mice.

\section{Materials and Methods}

Mouse Strains and Dissection

Expression profiling of $D k k 1$ was performed with wild-type embryos collected from timed matings of outbred Swiss Quackenbush (Affymetrix profiling) or CD1 (in situ hybridisation) mice. To assess the role of $D k k 1$ in gonad development, $D k k 1^{+/-}$ mice [Mukhopadhyay et al., 2001], maintained on a 129/C57BL6 background, were intercrossed. Noon of the day on which the mating plug was observed was designated $0.5 \mathrm{dpc}$. For more accurate staging, the tail somite (ts) stage of the embryo was determined by counting the number of somites posterior to the hind limb [Hacker et al., 1995]. Using this method, $11.5 \mathrm{dpc}$ corresponds to $\sim 18$ ts, and $12.5 \mathrm{dpc}$ to 28 ts. At $11.5 \mathrm{dpc}$, embryos were sexed by PCR for the Y-linked gene Zfy [Koopman et al., 1991]. From $D k k 1$-null litters, one urogenital ridge (UGR, gonad plus mesonephros) was dissected and fixed for immunofluorescence analysis; the other UGR was used for quantitative RT-PCR. Tail tissue from each embryo was used for genotyping as described [Mukhopadhyay et al., 2001], and all embryos genotyped as $D k k 1^{-1-}$ displayed craniofacial abnormalities consistent with the reported phenotype. All animal work was conducted according to protocols approved by the Animal Ethics and Care Committees of the University of Queensland and the Children's Medical Research Institute and the Children's Hospital at Westmead.

\section{Affymetrix Profiling}

Gonad pools at 11.0, 12.5 and $13.0 \mathrm{dpc}$ were used to produce probes for screening Affymetrix mouse 430v2 chips as detailed previously [Holt et al., 2006]. Experiments were run in triplicate and expression was calculated relative to $18 \mathrm{~S}$ rRNA. 
In situ Hybridisation

Whole-mount in situ hybridisation was carried out using standard methods [Hargrave et al., 2006]. The $D k k 1$ probe was supplied by Gudmap (Probe ID, 2178) (http://uqgudmap.imb.uq.edu. au/search_probe.phtml).

\section{Whole-Mount Immunofluorescence}

Immunofluorescence and image capture was performed as described [Combes et al., 2009]. Rabbit anti-SOX9 antibody has been described previously [Wilhelm et al., 2005] and was used at 1:200. Rat anti-VE-Cadherin (anti-vascular endothelial cadherin, BD Biosciences) was used at 1:200. Secondary antibodies (goat anti-rabbit Alexa Fluor 488 and anti-rat Alexa Fluor 594, Invitrogen) were used at 1:300.

\section{Quantitative Realtime RT-PCR}

Each UGR was processed and analysed individually. Total RNA was extracted and DNase-treated using an RNeasy Micro kit (Qiagen) as per manufacturers' instructions. cDNA was generated using a High-Capacity cDNA Archive kit (Applied Biosystems) according to manufacturers' instructions. Relative cDNA levels were analysed by the comparative cycle time $(\mathrm{Ct})$ method of quantitative RT-PCR (qRT-PCR) with reactions including Taqman PCR master mix (Applied Biosystems, ABI) and Taqman gene expression sets. Duplicate (Taqman) or triplicate (SYBR) assays were carried out on an ABI Prism 7000 Sequence Detector System, and the mean relative level of expression and associated standard deviations were calculated. Endogenous control, used to normalize gene expression levels, was Tbp (encoding TATA box binding protein). Taqman gene expression sets were as follows: Mm00446973_m1 (Tbp), Mm00443610_m1 (Axin2), Mm00500463_m1(Irx3),Mm00491634_m1(Sp5),Mm00437341_ m1 (Wnt4), Mm00431729_m1 (Dax1), Mm00507076_m1 (Rspo1), Mm00843544_s1 (Foxl2), Mm00514982_m1 (Follistatin), Mm01340178_m1 (Bmp2), Mm00448840_m1 (Sox9), Mm00442795_m1(Fgf9),Mm00432820_g1(Dhh),Mm00558507_ m1 (Cyp26b1), Mm03023963_m1 (Amh) and Mm00490735_m1 (Cyp11a1).

\section{Statistics}

Student's t tests (unpaired, 2-tailed) were used to determine statistical significance of expression differences between XY wild-type and XY null samples.

\section{Results}

Dkk1 Becomes Male-Specific in Its Expression Pattern Shortly after Sex Determination

Sry, the mammalian testis-determining gene, is expressed from 10.5 to $12.5 \mathrm{dpc}$ in mouse foetal gonads [Koopman et al., 1990]. In a microarray experiment designed to find novel genes expressed in a male- or femalespecific manner shortly after SRY triggers testis fate, we found that $D k k 1$ is upregulated at $12.0 \mathrm{dpc}$ in the developing testis, shortly after the upregulation of Sox9 (fig. 1A). By whole-mount in situ hybridisation (fig. 1B), low levels of $D k k 1$ expression were observed in the XY gonad at 11.5 $\mathrm{dpc}$ (solid arrow head). Expression was also observed in the paramesonephric duct (arrow) and mesonephric tubules (open arrowheads). At $12.5 \mathrm{dpc}$ in XY gonads, $D k k 1$ was expressed predominantly under the coelomic epithelium (arrowhead), whilst at $13.5 \mathrm{dpc}, D k k 1$ expression expanded to encompass the poles of the testis. $D k k 1$ was also expressed in the mesenchyme around the paramesonephric (müllerian) duct in 13.5-dpc XY samples (arrow) and could be related to the male-specific regression of this structure [Orvis and Behringer, 2007]. Dkk1 transcripts were not detected in 11.5- or 12.5-dpc XX gonads. At $11.5 \mathrm{dpc}$, mesonephric expression of $D k k 1$ in ovaries was similar to that observed in the testes. Differences emerged at $12.5 \mathrm{dpc}$ where $D k k 1$ expression was restricted to the dorsal end of the paramesonephric duct (arrows), more severely in XY compared to XX samples. Expression in mesonephric tubules also varied between the sexes (open arrowheads), and persisted in 13.5-dpc XX mesonephroi while it was not detected in XY samples of the same age. A single focal point of $D k k 1$ expression was consistently observed in the posterior region of the 13.5dpc ovary (arrowhead). Others have demonstrated a similar expression pattern of $D k k 1$ in wild-type XY and XX gonads by qRT-PCR, and demonstrated that expression of $D k k 1$ is first apparent in the central region of the $\mathrm{XY}$ coelomic epithelium at $12.5 \mathrm{dpc}$ [Manuylov et al., 2008].

\section{Ablation of Dkk1 Does Not Affect Expression of Key \\ Marker Genes of Gonadal Development}

In order to establish whether Dkkl has an important role during testis development, we examined the effect of loss of $D k k 1$ function on the expression of key markers of testis and ovarian development. We found little alteration in expression of such genes at $11.5 \mathrm{dpc}$ (online suppl. fig. 1; for all online supplementary material, see www. karger.com/doi/10.1159/000327709) or $12.5 \mathrm{dpc}$ (fig. 2). At $12.5 \mathrm{dpc}$, there was no upregulation of targets of the WNT canonical pathway (Axin2 and Sp5) [Jho et al., 2002; Clevers, 2006; Fujimura et al., 2007], suggesting that excessive WNT signalling is not occurring in the absence of $D k k 1$ (fig. 2A). Furthermore, there was no upregulation of Irx3, a WNT target gene that is highly expressed in the developing ovary [Braun et al., 2003; Jorgensen and Gao, 2005]. In addition, there was no molecular evidence of feminisation of the Dkk1-null XY gonad (fig. 2B) or significant difference in the expression of markers of testicular fate (fig. 2C). In summary, ablation of $D k k 1$ had no discernible effect on the early expression of key genes known to be associated with sex determination and gonad development. 


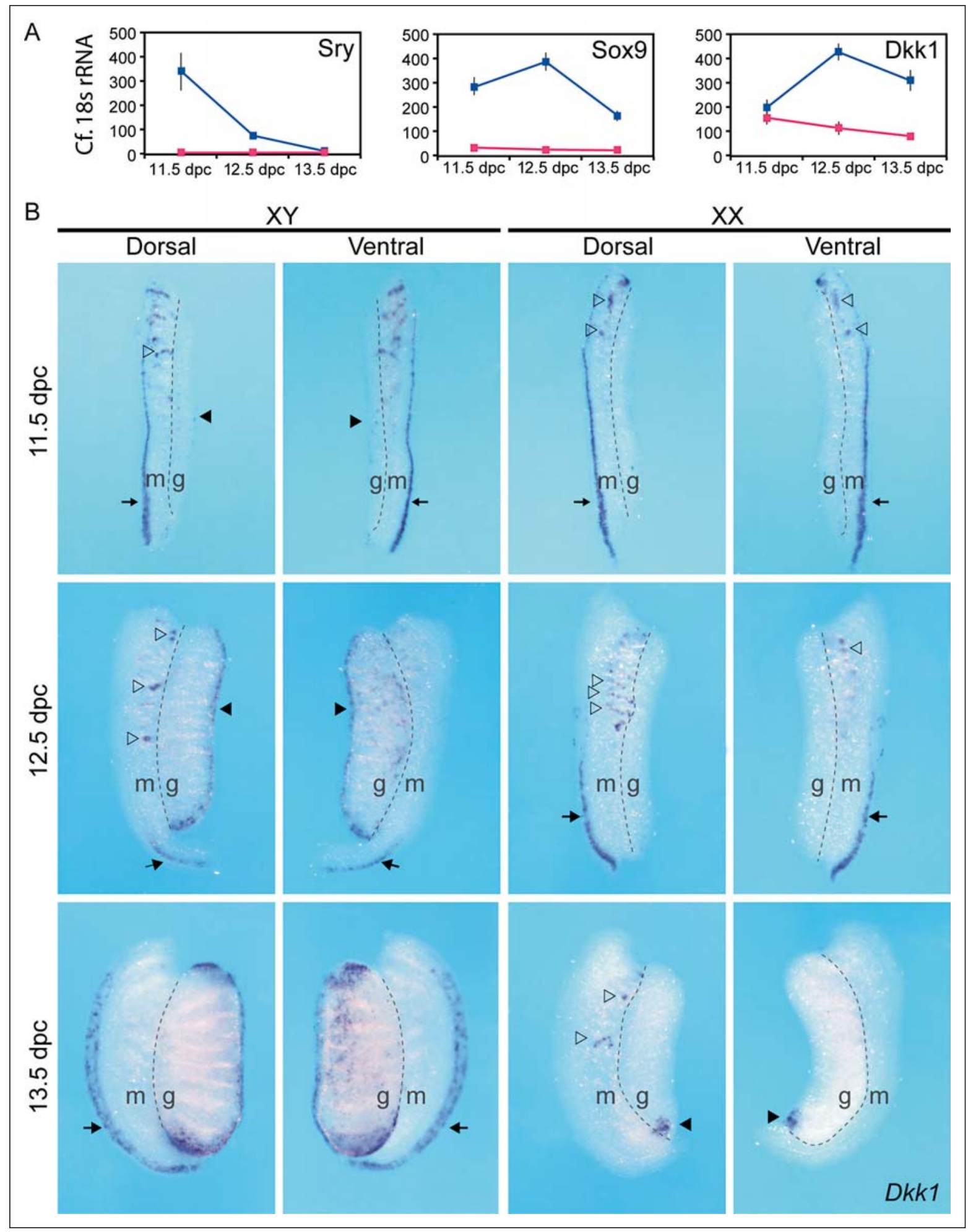

Fig. 1. $D k k 1$ is expressed in developing testis shortly after Sry and Sox9 are upregulated. A Expression of Sry (1450579_x_at), Sox9 (1434950_at) and Dkk1 (1420360_at), as detected by Affymetrix microarray screening. B Whole-mount in situ hybridisation of $D k k 1$ at $11.5,12.5$ and $13.5 \mathrm{dpc}$ in $\mathrm{XY}$ and $\mathrm{XX}$ urogenital ridges.
Both dorsal and ventral views are shown. $\mathrm{g}=$ Gonad; $\mathrm{m}=$ mesonephros; arrows = paramesonephric duct; open arrowheads = $D k k 1$ expression in mesonephric tubules; solid arrowheads = $D k k 1$ expression in gonads. 

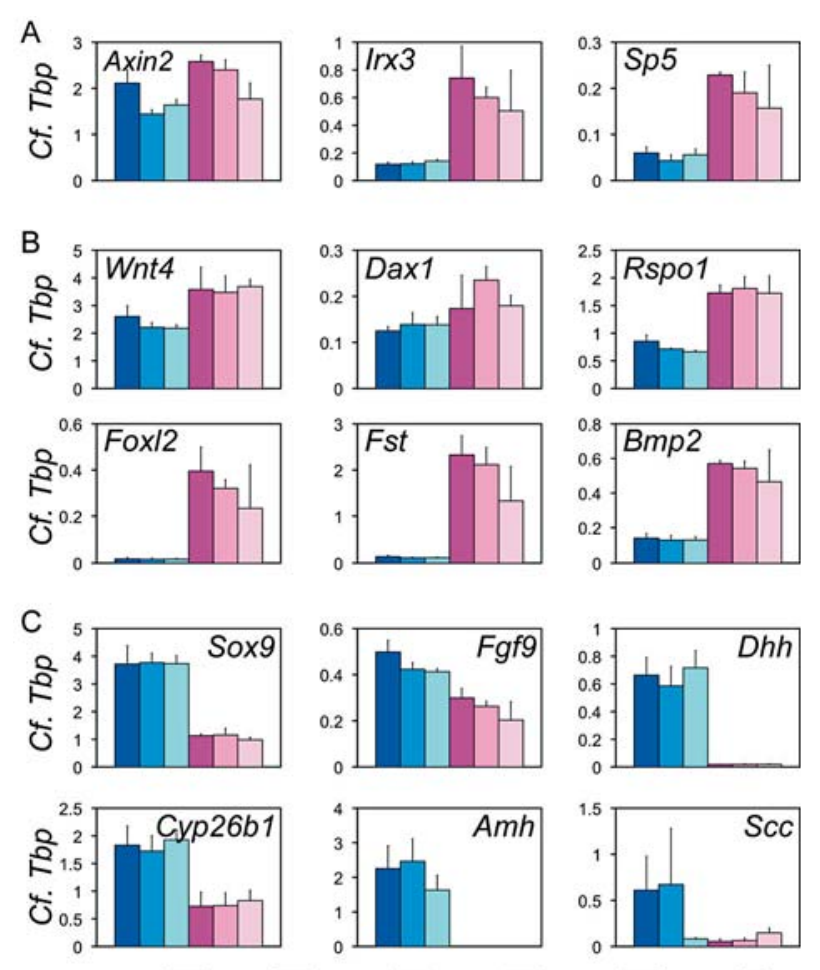

Legend XYWt XY het XYKO XXWt XX het $X X K O$

Fig. 2. Testis development in the Dkk1-null embryos appears normal with respect to expression of marker genes. A qRT-PCR analysis of transcriptional targets of the canonical WNT pathway, B markers of ovarian development, and $\mathbf{C}$ markers of testicular development in $12.5 \mathrm{dpc}$ UGR samples from wild-type (Wt), heterozygous (het) and null (KO) $D k k 1$ embryos. Bars indicate the mean \pm 1 SEM; $\mathrm{n}=5,3,5,2,4,3$. Endogenous control Tbp (encoding TATA box binding protein) was used to normalize gene expression levels.

\section{Ablation of Dkk1 Has Little Effect on Morphological} Development of the Foetal Testis

In view of the observation that loss of $D k k 1$ function has no effect on the core testis transcriptional program, we next investigated whether any developmental delay or disorganisation of testis development could be found in Dkkl-null XY gonads. Particular attention was given to the development of the male-specific coelomic blood vessel [Brennan et al., 2002], which assembles under the coelomic epithelium at $12.5 \mathrm{dpc}$, correlating with the restricted expression of $D k k 1 \mathrm{RNA}$ at this stage. Immunostaining results obtained using antibodies that mark
Sertoli cells (anti-SOX9) and germ cells/endothelial cells (anti-PECAM-1) revealed no delay or disruption of testis cord formation (fig. 3, asterisks). Further, endothelial cells that form the coelomic vessel localised to the appropriate area in $D k k 1$-null gonads and displayed a similar level of organisation to staged-matched controls (fig. 3, arrows).

\section{Discussion}

WNT signalling is known to be important in mouse and human ovarian development. The finding of a malespecific expression of a WNT antagonist, DKK1, shortly after testis fate is determined [Manuylov et al., 2008; present study], raises the possibility that DKK1 might play a role in modulating WNT signalling activity during gonadal development. As Wnt4 is expressed at early stages in both male and female gonads, it seemed plausible that the action of DKK1 might be critical in preventing WNT4-related feminisation activity in the XY gonads. However, our data show that ablation of $D k k 1$ has no apparent effect on mouse foetal testis development. Although we cannot rule out the possibility of a later phenotype arising, $\mathrm{WNT} / \beta$-catenin acts early, and if the absence of $D k k 1$ had an effect on sex determination, we would expect to see it in the time window studied. Two alternative explanations for these findings are redundancy of function between DKK1 and other WNT antagonists, or that DKK1 protects against ectopic WNT activity.

Although $D k k 1$ is a member of a multigene family, evidence for redundancy between DKK1 and proteins of related function in the gonad is not strong. Our microarray results suggest that $D k k 2, D k k 4$, Wif and Cerberus are not expressed at significantly higher levels in XY than in $\mathrm{XX}$ gonads and there is only a very modest upregulation of $D k k 3$ (online suppl. fig. 2). Male-specific expression of other (mechanistically different) antagonists of WNT signalling, Sfrp1 and Sfrp2 [Warr et al., 2009], may compensate for the lack of $D k k 1$. Testes are smaller and have fewer cords in compound mutants lacking Sfrp1 and Sfrp2 [Warr et al., 2009], leaving open the possibility of redundancy between $D k k 1$ and the Sfrp genes. However, this possibility remains to be tested by analysing doubleor triple-knockout mice.

Alternatively, the primary mechanism for WNT suppression in the testis may occur independent of signal inhibition. In this case, DKK1 could serve a protective role that is only apparent when challenged by ectopic 
Fig. 3. Testis development in the $D k k 1$-null embryos appears normal morphologically. A Immunofluorescent analysis of wholemount UGRs from wild-type $\left(D k k 1^{+/+}\right)$, B $D k k 1^{+/-}$, and C $D k k 1^{-/-}$embryos $(12.5$ dpc). SOX9 (green) marks Sertoli cells, PECAM (red) marks germ cells and vasculature. No delay or disruption of testis cord formation is seen (asterisks). Endothelial cells localise to the appropriate area in Dkk1-null gonads and display a similar level of organisation to staged-matched controls (arrows). Tail somite (ts) stages for each sample are indicated. Scale bar $=100$ $\mu \mathrm{m}$.
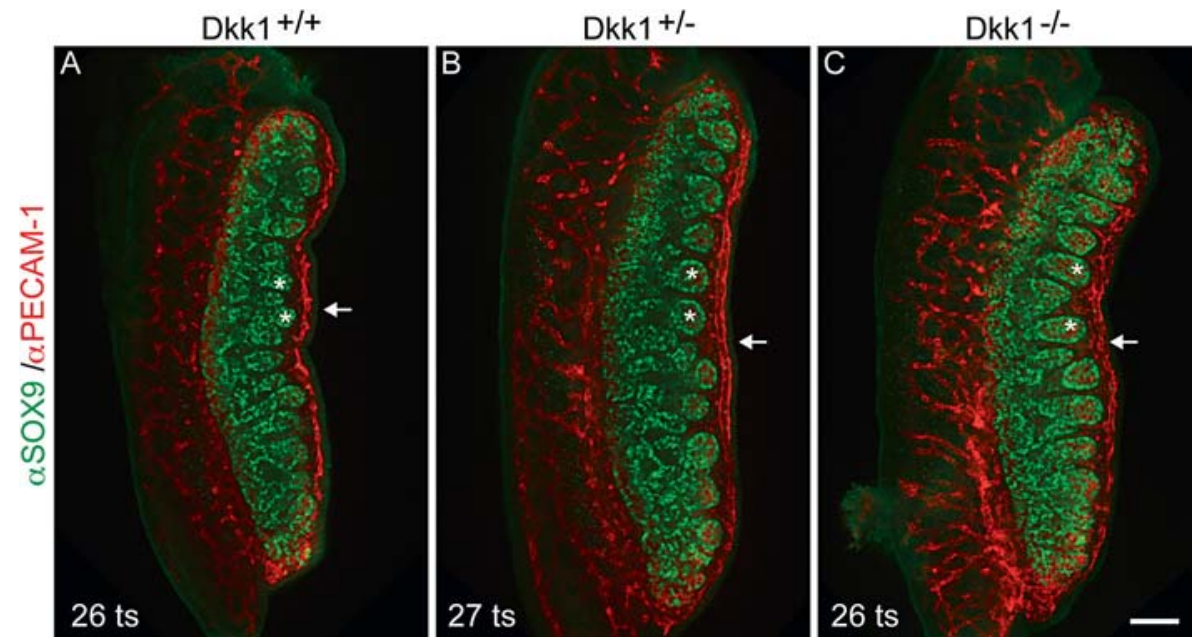

WNT activity. Further studies could test whether Dkk1null gonads are more susceptible to WNT-induced feminisation through culture with chemical agents such as lithium chloride [Bernard et al., 2008; Maatouk et al., 2008], or by studying available WNT4 overexpression mouse models [Jordan et al., 2001]. If DKK1 is found to play such a role, the $D k k 1$-null mouse model may prove useful in future mouse studies as a sensitized background to elucidate the molecular control of the female program of gonadal development underpinned by WNT signalling.

If signal inhibition is not the primary means of suppressing WNT signalling in the developing testis, then what is? There is a strong correlation between expression of the transcription factor SOX9 and cessation of WNT activity in the mouse foetal gonad. Overexpression of Sox9 is sufficient to suppress the ovarian program and masculinise XX gonads [Vidal et al., 2001], and conditional inactivation of this gene results in immediate upregulation of Wnt4 and Foxl2 in XY gonads [Barrionuevo et al., 2006]. However, no evidence has yet emerged for direct suppression of Wnt4 transcription by SOX9, which is conventionally viewed as a transcriptional activator. Further studies are required to understand the mechanism of SOX9 dominance over WNT activity, although at least part of this action is mediated by the secreted signalling molecule FGF9 [Kim et al., 2006].

Accumulating evidence suggests that gene dosages and expression dynamics vary substantially between mouse and human. Although our data indicate that DKK1 is not critical for mouse testis development, it remains possible that $D K K 1$ mutations underlie some cases of partial sex reversal or gonadal abnormality in humans, or that DKK1 may act as a genetic modifier of these conditions.

\section{Acknowledgements}

This work was supported by research grants from the Australian Research Council (ARC), National Health and Medical Research Council of Australia and the National Institutes of Health, USA (DK070136). Confocal microscopy was performed at the Australian Cancer Research Foundation Dynamic Imaging Centre for Cancer Biology.
References

Dkk1 in Gonadal Development
Barrionuevo F, Bagheri-Fam S, Klattig J, Kist R, Taketo MM, et al: Homozygous inactivation of Sox9 causes complete XY sex reversal in mice. Biol Reprod 74:195-201 (2006).

- Bernard P, Fleming A, Lacombe A, Harley VR, Vilain E: Wnt4 inhibits beta-catenin/TCF signalling by redirecting beta-catenin to the cell membrane. Biol Cell 100:167-177 (2008).

- Binnerts ME, Kim KA, Bright JM, Patel SM, Tran K, et al: R-spondin1 regulates Wnt signaling by inhibiting internalization of LRP6.
Proc Natl Acad Sci USA 104:14700-14705 (2007).

Braun MM, Etheridge A, Bernard A, Robertson $\mathrm{CP}$, Roelink H: Wnt signaling is required at distinct stages of development for the induction of the posterior forebrain. Development 130:5579-5587 (2003).

Brennan J, Karl J, Capel B: Divergent vascular mechanisms downstream of Sry establish the arterial system in the XY gonad. Dev Biol 244:418-428 (2002). 
Chassot AA, Ranc F, Gregoire EP, Roepers-Gajadien HL, Taketo MM, et al: Activation of beta-catenin signaling by Rspol controls differentiation of the mammalian ovary. Hum Mol Genet 17:1264-1277 (2008).

Clevers H: Wnt/beta-catenin signaling in development and disease. Cell 127:469-480 (2006).

-Combes AN, Wilhelm D, Davidson T, Dejana E, Harley V, et al: Endothelial cell migration directs testis cord formation. Dev Biol 326: 112-120 (2009).

Davidson G, Wu W, Shen J, Bilic J, Fenger U, et al: Casein kinase 1 gamma couples Wnt receptor activation to cytoplasmic signal transduction. Nature 438:867-872 (2005).

Ellwanger K, Saito H, Clement-Lacroix P, Maltry $\mathrm{N}$, Niedermeyer J, et al: Targeted disruption of the Wnt regulator Kremen induces limb defects and high bone density. Mol Cell Biol 28:4875-4882 (2008).

- Fujimura N, Vacik T, Machon O, Vlcek C, Scalabrin S, et al: Wnt-mediated down-regulation of Sp1 target genes by a transcriptional repressor Sp5. J Biol Chem 282:1225-1237 (2007).

-Hacker A, Capel B, Goodfellow P, Lovell-Badge $\mathrm{R}$ : Expression of Sry, the mouse sex determining gene. Development 121:1603-1614 (1995).

- Hargrave M, Bowles J, Koopman P: In situ hybridization of whole-mount embryos. Methods Mol Biol 326:103-113 (2006).

$\checkmark \mathrm{He}$ X, Semenov M, Tamai K, Zeng X: LDL receptor-related proteins 5 and 6 in Wnt/betacatenin signaling: Arrows point the way. Development 131:1663-1677 (2004).

-Heikkila M, Prunskaite R, Naillat F, Itaranta P, Vuoristo J, et al: The partial female to male sex reversal in Wnt-4-deficient females involves induced expression of testosterone biosynthetic genes and testosterone production, and depends on androgen action. Endocrinology 146:4016-4023 (2005).

Holt JE, Jackson A, Roman SD, Aitken RJ, Koopman P, McLaughlin EA: CXCR4/SDF1 interaction inhibits the primordial to primary follicle transition in the neonatal mouse ovary. Dev Biol 293:449-460 (2006).

-Jeays-Ward K, Hoyle C, Brennan J, Dandonneau M, Alldus G, et al: Endothelial and steroidogenic cell migration are regulated by Wnt4 in the developing mammalian gonad. Development 130:3663-3670 (2003).
Jeays-Ward K, Dandonneau M, Swain A: Wnt4 is required for proper male as well as female sexual development. Dev Biol 276:431-440 (2004).

Jho EH, Zhang T, Domon C, Joo CK, Freund JN, Costantini F: Wnt/beta-catenin/Tcf signaling induces the transcription of Axin2, a negative regulator of the signaling pathway. Mol Cell Biol 22:1172-1183 (2002).

Jordan BK, Mohammed M, Ching ST, Delot E, Chen XN, et al: Up-regulation of WNT-4 signaling and dosage-sensitive sex reversal in humans. Am J Hum Genet 68:1102-1109 (2001).

Jorgensen JS, Gao L: Irx3 is differentially up-regulated in female gonads during sex determination. Gene Expr Patterns 5:756-762 (2005).

Kim Y, Capel B: Balancing the bipotential gonad between alternative organ fates: A new perspective on an old problem. Dev Dyn 235: 2292-2300 (2006).

Kim Y, Kobayashi A, Sekido R, DiNapoli L, Brennan J, et al: Fgf9 and Wnt4 act as antagonistic signals to regulate mammalian sex determination. PLoS Biol 4:e187 (2006).

Koopman P, Munsterberg A, Capel B, Vivian N, Lovell-Badge R: Expression of a candidate sex-determining gene during mouse testis differentiation. Nature 348:450-452 (1990).

Koopman P, Ashworth A, Lovell-Badge R: The Zfy gene family in humans and mice. Trends Genet 7:132-136 (1991)

-Maatouk DM, DiNapoli L, Alvers A, Parker KL, Taketo MM, Capel B: Stabilization of betacatenin in XY gonads causes male-to-female sex-reversal. Hum Mol Genet 17:2949-2955 (2008).

Manuylov NL, Smagulova FO, Leach L, Tevosian SG: Ovarian development in mice requires the GATA4-FOG2 transcription complex. Development 135:3731-3743 (2008).

Mizusaki H, Kawabe K, Mukai T, Ariyoshi E, Kasahara M, et al: Dax-1 (dosage-sensitive sex reversal-adrenal hypoplasia congenita critical region on the $\mathrm{X}$ chromosome, gene 1 ) gene transcription is regulated by Wnt 4 in the female developing gonad. Mol Endocrinol 17:507-519 (2003).

Mukhopadhyay M, Shtrom S, Rodriguez-Esteban C, Chen L, Tsukui T, et al: Dickkopf1 is required for embryonic head induction and limb morphogenesis in the mouse. Dev Cell 1:423-434 (2001).
Nam JS, Turcotte TJ, Smith PF, Choi S, Yoon JK: Mouse Cristin/R-spondin family proteins are novel ligands for the Frizzled 8 and LRP6 receptors and activate beta-catenin-dependent gene expression. J Biol Chem 281: 13247-13257 (2006).

Orvis GD, Behringer RR: Cellular mechanisms of mullerian duct formation in the mouse. Dev Biol 306:493-504 (2007).

- Parma P, Radi O, Vidal V, Chaboissier MC, Dellambra E, et al: R-spondin 1 is essential in sex determination, skin differentiation and malignancy. Nat Genet 38:1304-1309 (2006).

-Tamai K, Zeng X, Liu C, Zhang X, Harada Y, et al: A mechanism for Wnt coreceptor activation. Mol Cell 13:149-156 (2004).

Tevosian SG, Manuylov NL: To beta or not to beta: Canonical beta-catenin signaling pathway and ovarian development. Dev Dyn 237: 3672-3680 (2008).

Tomizuka K, Horikoshi K, Kitada R, Sugawara Y, Iba Y, et al: R-spondin1 plays an essential role in ovarian development through positively regulating Wnt- 4 signaling. Hum Mol Genet 17:1278-1291 (2008)

-Vainio S, Heikkila M, Kispert A, Chin N, McMahon AP: Female development in mammals is regulated by Wnt-4 signalling. Nature 397: 405-409 (1999).

Vidal VP, Chaboissier MC, de Rooij DG, Schedl A: Sox9 induces testis development in XX transgenic mice. Nat Genet 28:216-217 (2001).

Warr N, Siggers P, Bogani D, Brixey R, Pastorelli L, et al: Sfrp1 and $S f r p 2$ are required for normal male sexual development in mice. Dev Biol 326:273-284 (2009).

-Wei Q, Yokota C, Semenov MV, Doble B, Woodgett J, He X: R-Spondin1 is a high affinity ligand for LRP6 and induces LRP6 phosphorylation and beta-catenin signaling. J Biol Chem 282:15903-15911 (2007).

Wilhelm D, Martinson F, Bradford S, Wilson MJ, Combes AN, et al: Sertoli cell differentiation is induced both cell-autonomously and through prostaglandin signaling during mammalian sex determination. Dev Biol 287:111-124 (2005).

-Yao HH, Matzuk MM, Jorgez CJ, Menke DB, Page DC, et al: Follistatin operates downstream of Wnt4 in mammalian ovary organogenesis. Dev Dyn 230:210-215 (2004).

Zeng X, Tamai K, Doble B, Li S, Huang H, et al: A dual-kinase mechanism for Wnt co-receptor phosphorylation and activation. Nature 438:873-877 (2005) 